without effect on photosystem II, but markedly stimulated the photoreduction of pyridine nucleotides by photosystem I. A recent report by Yocum and San Pietro suggests that this substance is not only essential to the photoreduction of nicotinamide adenine dinucleotide phosphate (NADP) by chloroplasts but is the primary electron acceptor at the reducing terminus of photosystem I (Biochem. Biophys. Res. Commun., 36, $614 ; 1969)$. This observation should satisfy anyone whose experiments have indicated the presence, if not the identity, of a chloroplast component with a considerably lower redox potential than ferredoxin. It seems that the "lipoidal factor" of Yocum and San Pietro is responsible for the reduction of ferredoxin and has thus been renamed "ferredoxin-reducing factor" (FRS).

Photochemical reactions in photosynthesis are concerned with the conversion of light energy into biochemical energy, stored in the energy-rich phosphate bonds of ATP, and with the generation of a source of reducing power in the form of reduced pyridine nucleotides (NADPH). This is achieved by the cooperation of two light reactions, coupled by series of electron carriers. Light of wavelengths shorter than $680 \mathrm{~nm}$ is trapped by the pigments of photosystem II and used to split water, providing a source of electrons, with the concomitant evolution of oxygen. Electrons from photosystem II are ferried to photosystem I by a series of intermediate electron carriers, and energy is conserved by the formation of ATP during this process. In experiments with chloroplast fragments photosystem II can be replaced as a source of electrons by an ascorbate/DPIP (2,6-dichlorophenolindophenol) couple, and plastocyanin. In these conditions, the photoreduction of NADP by photosystem I alone can be observed in vitro. Light of wavelengths greater than $680 \mathrm{~nm}$ is trapped by the pigments of photosystem I resulting in the ejection of an electron. This electron is replaced from photosystem II in vivo, or from the ascorbate/DPIP couple in vitro. The ejected electron is captured by the primary acceptor; at one time this was thought to be ferredoxin (redox potential= $-0.43 \mathrm{~V}$ at $p \mathrm{H} 7 \cdot 5)$. Experiments in which artificial electron acceptors, such as the viologen dyes, were reduced by chloroplasts lacking in ferredoxin indicated that another substance with a redox potential perhaps as low as $-0.55 \mathrm{~V}$ must be involved (for example, Kok et al., Biochim. Biophys. Acta, 109, 347; 1965). Yocum and San Pietro have demonstrated that their preparation of spinach chloroplast fragments will photoreduce NADP only if the reaction mixture contains an ascorbate/DPIP couple, plastocyanin, ferredoxin, ferredoxin-NADP reductase and ferredoxin-reducing substance. The rate of pyridine nucleotide reduction is much less if any of these requirements are omitted. Yocum and San Pietro propose that electrons produced by the action of light on photosystem I are used to reduce FRS. This, in turn, can reduce several artificial electron acceptors, but in vivo ferredoxin is the substrate. NADP is then reduced to NADPH by ferredoxin, the reaction being mediated by ferredoxin-NADP reductase.

In 1967, Fujita and Myers isolated a substance which they called "cytochrome-reducing factor" (CRS) (Arch. Biochem. Biophys., 119, 8; 1967). This substance resembles FRS in several properties; it catalyses the photoreduction of viologen dyes, but not the

photoreduction of NADP. The nature, identity and possible inter-relationship of CRS and FRS are being investigated; consideration of the speed with which significant advances in the photochemistry of photosynthesis have been made in recent years suggests that an early answer to the problem may be expected.

\title{
INVERTEBRATES
}

\section{Two New Squids}

These two squids, found in the Atlantic Ocean, have been described for the first time in $A$ Monograph of the Cephalopoda of the North Atlantic: The Family Cycloteuthidae, by Richard E. Young and Clyde F. E. Roper (Smithsonian, 40c). They belong to a new genus, Discoteuthis, and have been named $D$. discus $(a)$ and $D$. laciniosa $(b)$. Together with a related species, Cycloteuthis sirventi, they have been taken as the basis for a new family of cephalopods: the Cycloteuthidae. Their apparently frightening aspect is diminished by their small size; the conical mantle is $53 \mathrm{~mm}$ long in $D$. discus and $56 \mathrm{~mm}$ in D. laciniosa.

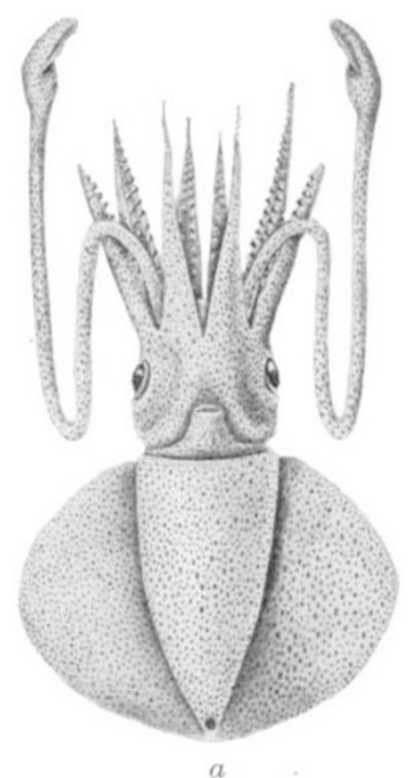

TEMPERATURE STANDARDS

\section{Measurement above the Gold Point}

An account of the recent work at the National Physical Laboratory on the establishment of temperature standards above the gold point $\left(1,064 \cdot 43^{\circ} \mathrm{C}\right)$ has now appeared in the Proceedings of the Royal Society (A,312, 31; 1969). What T. J. Quinn and M. C. Ford have done is a valuable complement to the optical standardization of temperatures above the gold point reported from the National Bureau of Standards in the United States by R. D. Lee (Metrologia, 2, 150; 1966). Although the sensitivities claimed for the two methods of temperature determination are substantially the same, that of Quinn and Ford may be more convenient because of its use of an especially stable secondary temperature standard-a black-body lamp consisting of a narrow tube of tungsten mounted in a glass envelope. 